\title{
Acute Rho-kinase inhibition improves coronary dysfunction in vivo, in the early diabetic microcirculation
}

\author{
James T Pearson ${ }^{1,2,3^{*}+}$, Mathew J Jenkins ${ }^{1,4+}$, Amanda J Edgley ${ }^{1,4}$, Takashi Sonobe ${ }^{5}$, Mandar Joshi ${ }^{6}$, \\ Mark T Waddingham ${ }^{1,4}$, Yutaka Fujii ${ }^{5}$, Daryl O Schwenke ${ }^{7}$, Hirotsugu Tsuchimochi ${ }^{5}$, Misa Yoshimoto ${ }^{5}$, \\ Keiji Umetani ${ }^{8}$, Darren J Kelly ${ }^{4}$ and Mikiyasu Shirai ${ }^{5}$
}

\begin{abstract}
Objectives: Activation of RhoA/Rho-kinase (ROCK) is increasingly implicated in acute vasospasm and chronic vasoconstriction in major organ systems. Therefore we aimed to ascertain whether an increase in ROCK activity plays a role in the deterioration of coronary vascular function in early stage diabetes.

Methods: Synchrotron radiation microangiography was used to determine in vivo coronary responses in diabetic (3 weeks post streptozotocin $65 \mathrm{mg} / \mathrm{kg}$ ip) and vehicle treated male Sprague-Dawley rats ( $n=8$ and 6 ). Changes in vessel number and calibre during vasodilator stimulation before and after blockade of nitric oxide synthase and cyclooxygenase were compared between rats. Acute responses to ROCK inhibitor, fasudil (10 mg/kg iv) was evaluated. Further, perivascular and myocardial fibrosis, arterial intimal thickening were assessed by histology, and capillary density, nitrotyrosine and ROCK1/2 expressions were evaluated by immunohistochemical staining.

Results: Diabetic rats had significantly elevated plasma glucose $(P<0.001$ vs control), but did not differ in fibrotic scores, media to lumen ratio, capillary density or baseline visible vessel number or calibre. Responses to acetylcholine and sodium nitroprusside stimulation were similar between groups. However, in comparison to control rats the diabetic rats showed more segmental constrictions during blockade, which were not completely alleviated by acetylcholine, but were alleviated by fasudil. Further, second order vessel branches in diabetic rats were significantly more dilated relative to baseline $(37 \%$ vs $12 \%$ increase, $P<0.05)$ after fasudil treatment compared to control rats, while visible vessel number increased in both groups. ROCK2 expression was borderline greater in diabetic rat hearts $(P<0.053)$.

Conclusions: We found that ahead of the reported decline in coronary endothelial vasodilator function in diabetic rats there was moderate elevation in ROCK expression, more widespread segmental constriction when nitric oxide and prostacyclin production were inhibited and notably, increased calibre in second and third order small arteries-arterioles following ROCK inhibition. Based on nitrotyrosine staining oxidative stress was not significantly elevated in early diabetic rats. We conclude that tonic ROCK mediated vasoconstriction contributes to coronary vasomotor tone in early diabetes.
\end{abstract}

\section{Introduction}

Diabetes is associated with coronary microvascular dysfunction due to an inability of the endothelium to maintain vasodilatory tone both at rest [1] and during stress [2,3]. This previous work has primarily focused on later timepoints of diabetes, where microvascular damage is already

\footnotetext{
* Correspondence: james.pearson@monash.edu

${ }^{\dagger}$ Equal contributors

'Department of Physiology, Monash University, Melbourne, Australia

${ }^{2}$ Monash Biomedical Imaging Facility, Melbourne, Australia

Full list of author information is available at the end of the article
}

well developed and thus difficult to reverse. As diabetes progresses these individuals are subjected to a vastly increased risk of ischaemic heart disease, acute myocardial infarction, and stroke $[4,5]$. Utilising synchrotron radiation (SR) microangiography, we have now demonstrated that even in the early stages of diabetes in the coronary circulation focal and segmental constrictions occur when prostacyclin and nitric oxide contribution is prevented, although globally basal endothelium-dependent vasodilation is maintained [6]. This impairment in vasodilatory capacity may 
contribute to the increased vulnerability to ischemia and myocardial infarction observed in advanced diabetes. Thus, it is of paramount importance that the mechanisms underlying this localised dysfunction are further elucidated, at a time point where potential intervention remains possible.

One pathway that is increasingly recognised to be involved in the pathogenesis of cardiovascular disease is the RhoA/ROCK pathway, which has been implicated in the progression of conditions including hypertension [7], stroke [8], coronary vasospasm and angina $[9,10]$, ischemiareperfusion injury and heart failure $[11,12]$. RhoA is a small plasma membrane bound guanosine-5'-triphosphatebinding protein, which when stimulated, activates ROCK [12]. It is thought that upregulation of RhoA/ROCK in the diabetic vasculature [13] causes subsequent phosphorylation of downstream signalling targets including myosin light chain phosphatase, and increased constriction of vascular smooth muscle [14-16]. Thus there may be a role for ROCK in the early diabetic coronary dysfunction we have previously described [6].

Acute treatment with ROCK inhibitors has shown promising results, with reduction in ischaemic damage [15,17-19], improvement in cerebral vasodilation in type II diabetic mice [20] and decreased vascular resistance and increased peripheral blood flow in patients with heart failure [11]. Notably, ROCK inhibitors may also have beneficial outcomes in preventing the development of coronary dysfunction, most likely by promoting or maintaining increased expression and activity of vasoprotective endothelial nitric oxide synthase (NOS) [14,21]. This study therefore aimed to ascertain whether ROCK plays a role in the deterioration of coronary vascular function in early stage diabetes.

\section{Methods}

\section{Animals and experiments at the synchrotron}

Experiments were conducted at SPring-8, Japan Synchrotron Radiation Research Institute, Hyogo, Japan with approval from the Animal Experiment Review Committee in accordance with the guidelines of the Physiological Society of Japan. Male Sprague Dawley rats (Japan SLC, Kyoto, Japan, 7 wks old) received either a vehicle injection of sodium citrate $(0.1 \mathrm{M}, \mathrm{pH} 4)$ (control) or streptozotocin (STZ; $65 \mathrm{mg} / \mathrm{kg}$ i.p) to induce type I diabetes. All rats were on a $12 \mathrm{hr}$ light/dark cycle at $18-25^{\circ} \mathrm{C}$ and were provided with food and water ad libitum. Three weeks after vehicle or STZ injection all rats underwent terminal angiography experiments. Fasted conscious blood glucose was measured via the tail vein two days prior to imaging to minimise stress.

\section{Experimental preparation}

Under sodium pentobarbital anaesthesia (50 mg/kg i.p.), rats were intubated, artificially ventilated (Shinano, Tokyo,
Japan; 40\% oxygen) and the right carotid artery cannulated with a radiopaque 20-gauge BD Angiocath catheter (Becton Dickinson, Inc., Sandy, Utah, USA), placing the tip at the entrance of the aortic valve. Body temperature was maintained at $37^{\circ} \mathrm{C}$, using a rectal thermistor coupled with a thermostatically controlled heating pad. Anaesthesia level was maintained via additional intraperitoneal boluses of pentobarbital (25 mg/kg/h). Sodium lactate (Sigma-Aldrich Japan K.K., Tokyo, Japan) was administered intravenously via the right jugular vein to maintain body fluids $(3.0 \mathrm{ml} /$ hr). A $0.3 \mathrm{ml}$ sample of venous blood was collected in EDTA coated tubes, centrifuged at $4^{\circ} \mathrm{C}$ for $15 \mathrm{~min}$ at $5000 \mathrm{rpm}$ and plasma removed for storage at $-20^{\circ} \mathrm{C}$ until subsequent triglyceride concentration determination (SRL Inc., Tachigawa, Tokyo, Japan). A catheter filled with heparinised saline (12 units/ml), was inserted into the right femoral artery to record arterial pressure via a pressure transducer (MLT0699, AD Instruments, NSW, Australia) using CHART software (v5.4.1, AD Instruments, NSW, Australia) to determine mean arterial pressure (MAP) and heart rate (HR), simultaneous with recordings of the camera trigger over the cardiac cycle.

\section{Angiography protocol}

Each rat was then placed in line with the horizontal X-ray beam and SATICON detector system (Hitachi Denshi Techno-system, Ltd., Tokyo, Japan and Hamamatsu Photonics, Shizuoka, Japan), as described previously [6]. Pancuronium bromide (Mioblock; $2 \mathrm{mg} / \mathrm{kg}$, Sankyo, Tokyo, Japan,) was administered for neuromuscular blockade to prevent spontaneous breathing when artificial ventilation was briefly stopped during imaging. Iodinated contrast medium (Iomeron 350; Bracco-Eisai Co. Ltd, Tokyo, Japan) was injected intrarterially as a bolus $(0.3-0.5 \mathrm{ml}$ at $0.4 \mathrm{ml} / \mathrm{s}$ ) into the aorta with a clinical autoinjector (Nemoto Kyorindo, Tokyo, Japan) at the start of image recording scans. At least 10 minutes was allowed for renal clearance of contrast between imaging scans. During each cine-scan, monochromatic X-rays at $33.2 \mathrm{keV}$ (energy bandwidth $20-30 \mathrm{eV}$ ) and a flux $\sim 10^{10}$ photons $/ \mathrm{mm}^{2} / \mathrm{s}$, passed through the rats chest and were recorded on the SATICON detector at $30 \mathrm{frames} / \mathrm{s}$ at 10-bit resolution for $\sim 3 \mathrm{~s}$ intervals. For each cine-scan, 100 frames were recorded with a short shutter open time of $1.5-2.0 \mathrm{~ms} /$ frame and a $9.5 \mu \mathrm{m}$ equivalent pixel size for the $9.5 \times 9.5 \mathrm{~mm}$ input field with images stored in $1024 \times 1024$ pixel format.

\section{Experimental protocol}

Endothelium-dependent and -independent vasodilatory responses were recorded in control $(n=6)$ and diabetic $(\mathrm{n}=8)$ animals. Angiogram series were recorded at the end of 5 minute infusions of vehicle (sodium lactate $3.0 \mathrm{ml} / \mathrm{hr})$, ACh $(3.0 \mu \mathrm{g} / \mathrm{kg} / \mathrm{min})$, sodium nitroprusside (SNP $3.0 \mu \mathrm{g} / \mathrm{kg} / \mathrm{min}$ ), during vehicle infusion 30 minutes 
after combined inhibition of nitric oxide and prostacyclin production with $\mathrm{N} \omega$-nitro-l-arginine methyl ester ( $\mathrm{L}$ NAME, $10 \mathrm{mg} / \mathrm{kg}$ iv. bolus) and sodium meclofenamate ( $2 \mathrm{mg} / \mathrm{kg}$ iv. bolus) respectively. For simplicity, combined blockade refers to L-NAME + meclofenamate treatment together. Endothelium-dependent vasodilation was then assessed during combined blockade with a repeat infusion of $\mathrm{ACh}(3.0 \mu \mathrm{g} / \mathrm{kg} / \mathrm{min})$. A final image series was recorded 10 minutes after administration of fasudil hydrochloride $(10 \mathrm{mg} / \mathrm{kg}$ iv. ROCK inhibitor, HA1077, Tocris) [22]. Hence, all rats were imaged during 6 consecutive treatment periods in total.

\section{Tissue collection histology and immunohistochemistry}

Hearts were fixed in 10\% neutral buffered formalin and stored in 70\% ethanol. All histological and immunohistochemical sections were imaged using the Aperio ScanScope XT Slide Scanner (Aperio Technologies, Inc., CA, USA) system. The proportional area of the stained protein was automatically quantified using the Positive Pixel Count v9 algorithm on Aperio Imagescope (v11.0.2.725, Aperio Technologies). Non-round vessels, resulting from oblique transection or branching, were excluded from quantification of fibrosis and media-to-lumen ratio.

In $4 \mu \mathrm{m}$ thick sections of LV the vessel media-tolumen ratio (the area of the vessel media wall divided by the area of the total blood vessel lumen) was calculated [21]. Myocardial interstitial and perivascular fibrosis was assessed using picrosirius red stained LV sections [6]. Perivascular fibrosis was evaluated around coronary arterioles, as the ratio of the area of fibrosis immediately surrounding the intramyocardial blood vessel walls to the total area of the vessel [21].

Capillary density in the myocardium was detected as the proportion of positively stained endothelial cells with murine-specific endothelial cell marker isolectin B4 (1:50, Vector Laboratories, Inc, Burlingame, CA, USA) [23]. Briefly, after dewaxing and heat-mediated antigen retrieval, nonspecific protein binding was blocked with $20 \%$ normal goat serum (Dako, Golstrup, Denmark). Sections were then incubated with biotinylated isolectin $\mathrm{B} 4$ at $4^{\circ} \mathrm{C}$ over-night, followed by avidin-biotin horseradish peroxidase (Vector Laboratories, Inc, Burlingame, CA, USA) and diaminobenzidine (Vector Laboratories, Inc, Burlingame, CA, USA), as described previously [23].

ROCK1/2 and nitrotyrosine staining was performed after subjecting sections to heat-mediated antigen retrieval, followed by incubation with $3 \% \mathrm{H}_{2} \mathrm{O}_{2}$ for $15 \mathrm{~min}$ at room temperature and washing three times with PBS ( $\mathrm{pH}$ 7.4) for $5 \mathrm{~min}$ each. Nonspecific protein binding was blocked with 20\% normal goat serum (Dako, Golstrup, Denmark) for $30 \mathrm{~min}$. The sections were then incubated with the primary antibody overnight at $4^{\circ} \mathrm{C}$ (ROCK1 1:200 dilution, and ROCK2 1:250 dilution, Abcam, Cambridge,
USA; anti-nitrotyrosine 1:400, Millipore 6-284). Following this, sections were incubated with goat anti-rabbit horseradish peroxidase (Dako, Golstrup, Denmark) for 40 or $60 \mathrm{~min}$ (ROCK1 and ROCK2/nitrotyrosine respectively) at room temperature and developed using diaminobenzidine (Vector Laboratories, Inc, Burlingame, CA, USA) and finally counter-stained with haematoxylin.

\section{Assessment of vessel ID}

Vessel ID in individual rats was assessed as previously described [6]. Briefly, quantitative analysis of vessel ID was based on measurements from the middle of discrete vessel segments in individual cine-radiogram frames using ImageJ (v1.41, NIH, Bethesda, USA) for individual rats during each treatment period. Angiograms shown in this paper underwent median filtering (2 pixel radius) to improve vessel clarity for publication purposes only. Arterial vessels were categorised according to their branching order and their basal vessel ID size class (40-100 $\mu \mathrm{m}, 100-200 \mu \mathrm{m}$, 200-300 $\mu \mathrm{m}$ and $>300 \mu \mathrm{m})$. Reported results for vessel ID and vessel number in each rat during drug infusions are expressed as percentage change from baseline $(\Delta)$, to account for differences in absolute baseline vessel ID and vessel number between groups. Vessel recruitment was determined as the change in vessel number from baseline during each treatment for the same field of view.

\section{Quantification of segmental vasoconstrictions}

Relative change in vessel calibre following vasodilator inhibition gives no indication of the number of vessels with calibres less than the individual's mean change. Therefore the number of segmental vasoconstrictions during treatment periods was quantified during the treatment periods as outlined by Jenkins et al. [6]. Localised segmental vasoconstrictions were considered to be present when most of the length of a vessel segment $(<100 \mu \mathrm{m})$ showed an ID constriction of $>30 \%$ of baseline vessel ID.

\section{Statistical analysis}

Data is expressed as mean \pm SEM unless otherwise stated. The mean vessel ID and the change in ID (\%) of each branching order or vessel size class in individual rats, in each treatment period, were pooled for group comparisons. One-way and two-way ANOVA with Bonferroni correction for repeated measures was performed to assess within and between group differences due to treatments. Following ANOVA, between group comparisons were made using a 2-tailed Student t-test. The Statistical Package Software System (SPSS v15, SPSS Inc, Chicago, USA) was used for all analysis with values of $P<0.05$ deemed significant. 


\section{Results}

Animal characteristics

Diabetic animals had higher blood glucose concentrations $(P<0.001)$, lower final body weight $(P<0.001)$ and reduced heart rate under anaesthesia $(P<0.001)$ versus controls (Table 1 ). Kidney to BW ratio was significantly increased in diabetic animals $(P<0.05)$ while $\mathrm{LV}$ to $\mathrm{BW}$ ratio was comparable to control animals, indicating that there was no compensatory cardiac hypertrophy. Triglyceride concentration was similar in both groups.

\section{Structural changes and oxidative stress}

Myocardial perivascular fibrosis was similar in diabetic and control rats (Figure $1 \mathrm{~A}-\mathrm{C}$ ), as was media-to-lumen ratio (Figure 1D-F). There was also no significant difference in myocardial interstitial fibrosis between control and diabetic animals (Figure 2A-C) or capillary density (Figure 2D-F). Myocardial ROCK1 expression trended higher in diabetic versus control animals (Figure 3A-C), while myocardial ROCK2 expression was borderline significantly higher in diabetic animals 3 -weeks post STZ treatment compared to controls $(P<0.053$, Figure 3D-F). Nitrotyrosine levels did not differ significantly between groups, suggesting that diabetic rats did not experience consistently higher levels of oxidative stress (Figure 4).

\section{Baseline vessel internal diameter}

Representative angiogram frames for all treatment periods are presented in Figures 5 and 6 for a control and STZ rats to illustrate the limited individual variability in responses. Basal vessel ID in diabetic animals was comparable to control vessel ID across first ( $214 \pm 21$ vs. $224 \pm 18 \mu \mathrm{m})$, second $(141 \pm 8$ vs. $127 \pm 6 \mu \mathrm{m})$ and third (106 \pm 6 vs. $93 \pm 5 \mu \mathrm{m})$ order arterial vessels (Figure 7 ). Mean total visible vessel branching segments per animal in the field of view, was 12-18 vessels across all treatments in control animals and a comparable 11-14 vessels in diabetic rats. The minimum visualised vessel ID recorded across both groups was $44 \mu \mathrm{m}$.

Table 1 Body and organ weights, metabolic profile and haemodynamic variables in anaesthetised control and diabetic Sprague-Dawley rats

\begin{tabular}{lccc}
\hline & Control & Diabetes & $\boldsymbol{P}$ \\
\hline Body Weight (BW, g) & $312 \pm 10$ & $236 \pm 8$ & $<0.001$ \\
Blood Glucose (mmol) & $5.9 \pm 0.4$ & $19.5 \pm 1.9$ & $<0.001$ \\
MAP (mmHg) & $116 \pm 6$ & $105 \pm 7$ & NS \\
Heart Rate (bpm) & $436 \pm 7$ & $344 \pm 11$ & $<0.001$ \\
Triglyceride (mmol) & $0.57 \pm 0.05$ & $0.48 \pm 0.21$ & NS \\
Heart:BW (\%) & $0.267 \pm 0.005$ & $0.272 \pm 0.005$ & NS \\
Kidney:BW (\%) & $0.532 \pm 0.019$ & $0.820 \pm 0.023$ & $<0.001$ \\
\hline
\end{tabular}

Values expressed as mean \pm SEM. Control, $n=6$ and diabetic, $n=8$. MAP: Mean arterial pressure.

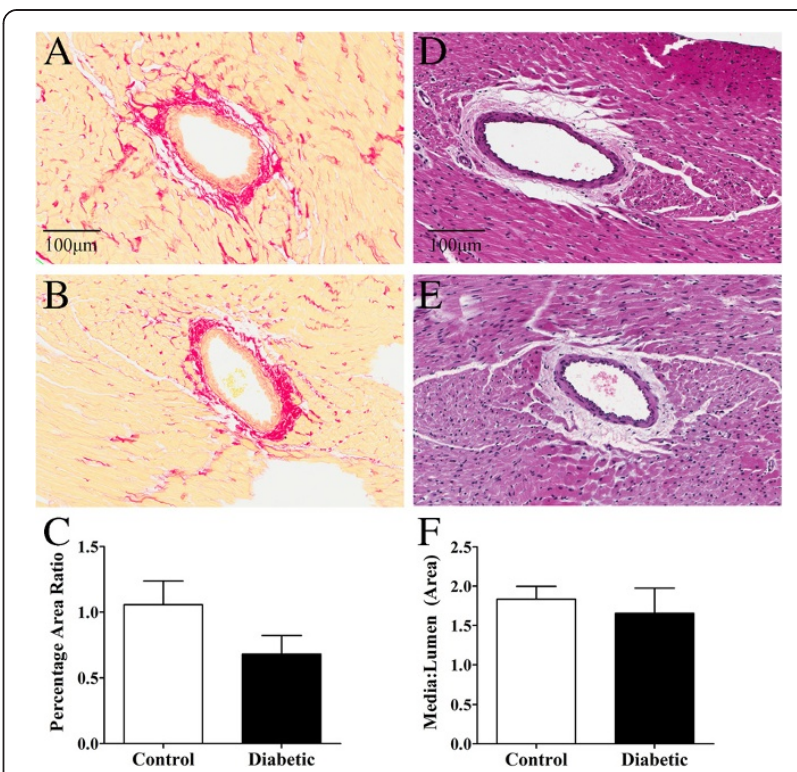

Figure 1 Myocardial perivascular fibrosis and media:lumen ratio in control and diabetic rats. Perivascular collagen ratio (C) was assessed via picrosirius red staining in control $(\mathbf{A})$ and diabetic $(\mathbf{B})$ rats (20x objective). Vessel media-to-lumen ratio (F) was assessed via haematoxylin and eosin staining in control (D) and diabetic (E) rats (20x objective). There was no significant difference between groups. Control, $n=6$ and diabetic, $n=8$. Values expressed as mean \pm SEM.
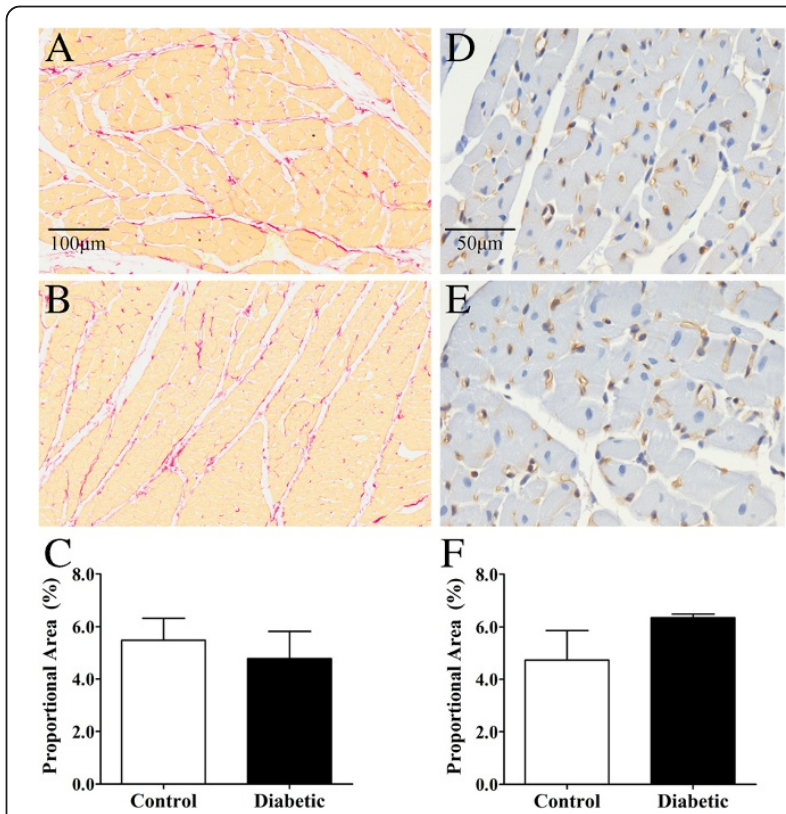

Figure 2 Myocardial interstitial collagen expression and capillary density in control and diabetic rats. Interstitial collagen accumulation $(\mathbf{C})$ assessed via picrosirius red staining in control (A) and diabetic (B) rats (20x objective). Capillary density (F) assessed via isolectin B4 staining in control (D) and diabetic (E) rats

(40x objective). There was no significant difference between groups. Control, $n=6$ and diabetic, $n=8$. Values expressed as mean \pm SEM. 


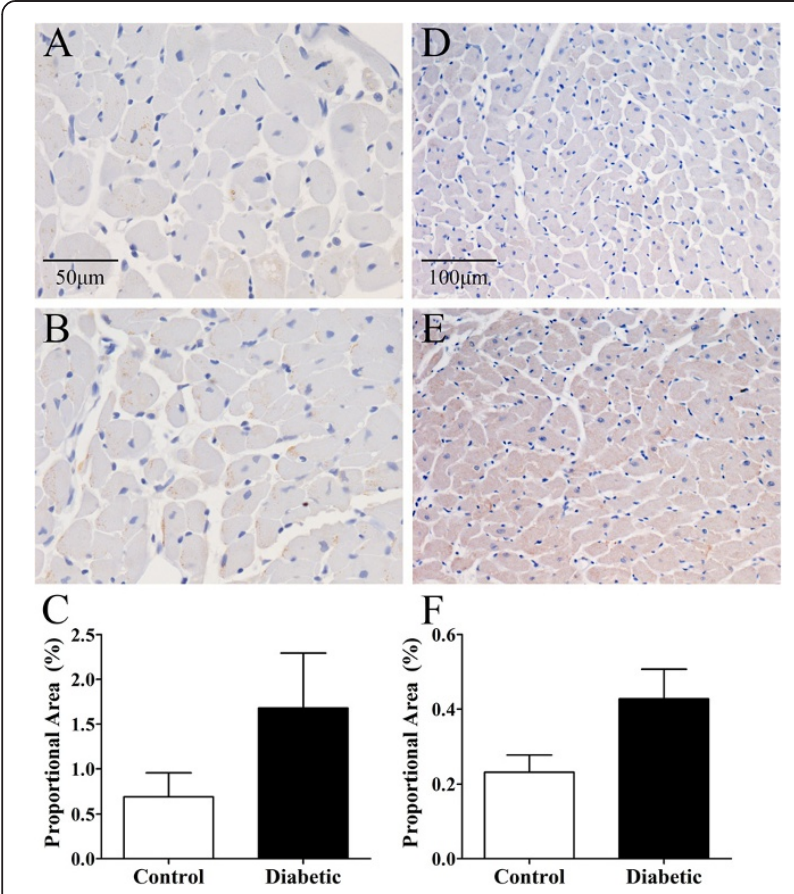

Figure 3 Myocardial ROCK1 and ROCK2 immunohistochemistry in control and diabetic rats. ROCK1 expression (C) in control (A) and diabetic (B) rats (40X objective). ROCK2 expression (F) in control (D) and diabetic (E) rats (20x objective). ROCK1 expression was not significantly different between control and diabetic groups, although there was a trend towards increased ROCK1 expression in diabetic rodents. ROCK2 expression was borderline significantly increased $(P<0.053)$ in diabetic compared to control animals. Control, $n=6$ and diabetic, $n=7$. Values expressed as mean \pm SEM.

\section{Vessel response to endothelium-dependent} and -independent stimulation

Basal endothelium-dependent vasodilation in diabetics in response to $\mathrm{ACh}$ was on average more pronounced ( $>40 \%$ of basal ID) than in control rats, but not significantly so, based on vessel order or vessel class (Figure 8A and $\mathrm{F}$ ). In response to $\mathrm{ACh}$, similar reductions in MAP $(\Delta-23.3 \pm 6.0 \%$ vs. $-31.4 \pm 6.5 \%)$ and HR $(\Delta-6.87 \pm 3.09 \%$ vs. $-2.21 \pm 2.38 \%$, Figure 9 ) were observed in both groups. Basal endothelium-independent dilatory responses to SNP were comparable between diabetic and control animals (Figure $8 \mathrm{~B}$ and $\mathrm{G}$ ). In addition, the MAP and HR response to SNP was not significantly different between groups (Figure 9).

\section{Vessel ID response during vasodilator inhibition}

Combined blockade of NOS and cyclooxygenase (COX) caused vasodilation of the coronary vasculature in both control and diabetic animals. This was evidenced by a slightly greater vessel ID in both groups compared to baseline (Figure $8 \mathrm{C}$ and $\mathrm{H}$ ). However, diabetic animals had a slightly blunted, but not significant, increase in MAP in response to NOS/COX blockade $(\Delta 9.7 \pm 9.7 \%$
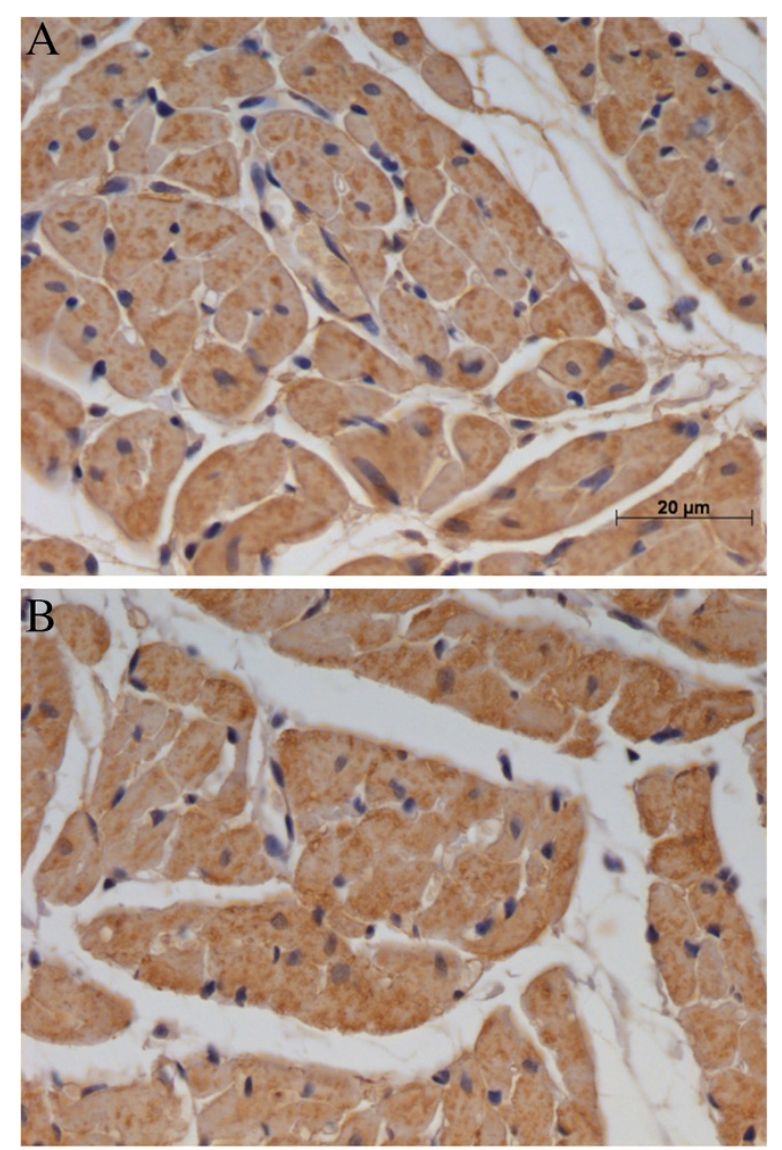

C

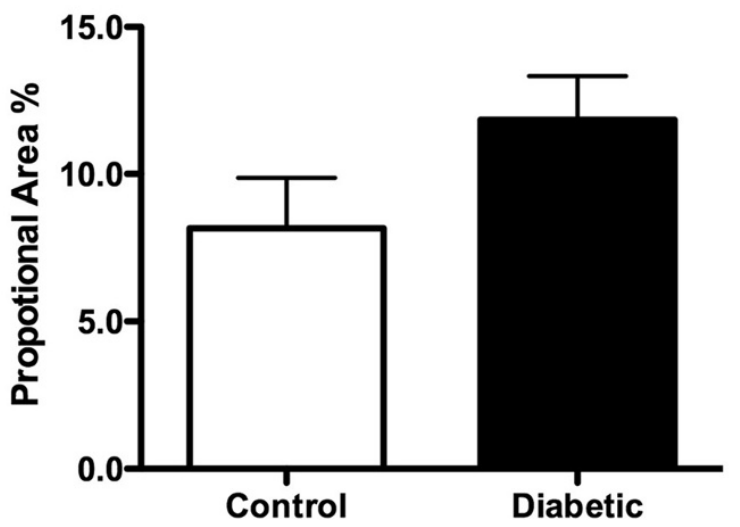

Figure 4 Myocardial nitrotyrosine immunohistochemistry in control and diabetic rats. Nitrotyrosine expression (C) in control (A) and diabetic (B) rats (20x objective). Nitrotyrosine expression was not significantly different between control and diabetic groups Control, $n=5$ and diabetic, $n=7$. Values expressed as mean \pm SEM.

vs. $31.9 \pm 16.4 \%$, Figure 9). HR was reduced by similar amounts in control and diabetic animals following NOS/ COX blockade. Vessel ID response to ACh stimulation during NOS/COX blockade was not different between control and diabetic rats (Figure $8 \mathrm{D}$ and I). Further, MAP in control and diabetic animals returned to baseline levels, 


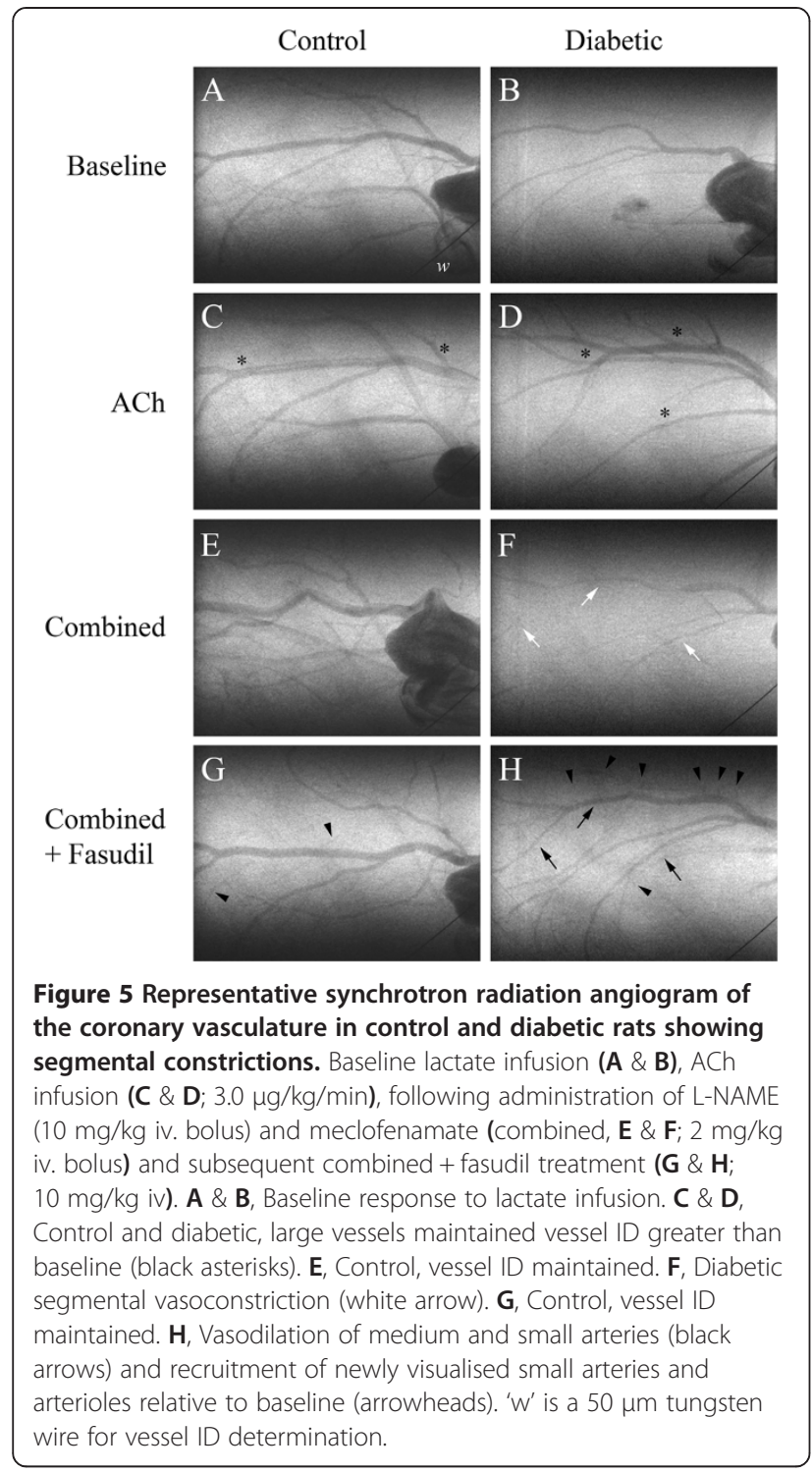

while there was a small but persistent reduction in HR in both groups (Figure 9).

\section{Vessel ID response to fasudil treatment}

Inhibition of ROCK during NOS/COX blockade resulted in a significantly greater vessel ID response in second order vessels in diabetic animals compared to controls $(\Delta 36.7 \pm 8.5 \%$ vs. $12.4 \pm 5.0 \%, P<0.05$, Figure $8 \mathrm{E})$. This difference in vessel ID, based on branching order, is also borne out when vessels were categorised by vessel class, as fasudil treatment caused a marked increase in vessel ID in 40-100 $\mu \mathrm{m}$ vessels from diabetic animals compared to controls $(P<0.05$, Figure 8J). There was a trend in both control and diabetic groups towards a progressively greater vasodilatory response to fasudil as vessel size class decreased. The greater increase in microvessel ID in diabetic animals did not correspond with a significantly greater

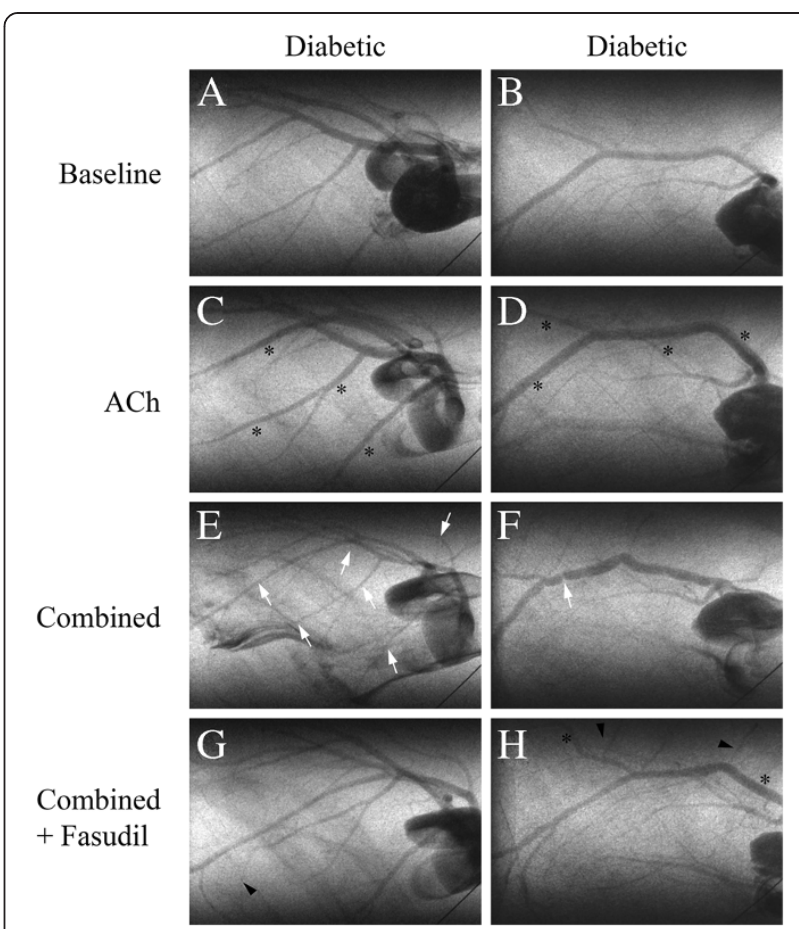

Figure 6 Additional synchrotron radiation angiograms of the coronary vasculature in two diabetic rats showing segmental constrictions. Baseline lactate infusion (A \& B), ACh infusion (C \& D; $3.0 \mu \mathrm{g} / \mathrm{kg} / \mathrm{min})$, following administration of L-NAME (10 mg/kg iv. bolus) and meclofenamate (combined, $\mathbf{E} \& \mathbf{F} ; 2 \mathrm{mg} / \mathrm{kg}$ iv. bolus) and subsequent combined + fasudil treatment ( $\mathbf{G} \& \mathbf{H} ; 10 \mathrm{mg} / \mathrm{kg} \mathrm{iv}$ ).

$\mathbf{A} \& \mathbf{B}$, Baseline response to vehicle infusion. $\mathbf{C} \& \mathbf{D}$, Both diabetics maintained larger vessel ID than baseline (black asterisks).

$\mathbf{E} \& \mathbf{F}$, Diabetic focal and segmental vasoconstrictions (white arrows). $\mathbf{G} \& \mathbf{H}$, Restoration of medium and small arteries to baseline ID, dilation (black asterisks) and recruitment of newly visualised small arteries and arterioles relative to baseline (arrowheads). ' $W$ ' is a $50 \mu \mathrm{m}$ tungsten wire for vessel ID determination.

reduction in MAP from baseline in comparison to control rats $(\Delta-51.8 \pm 2.6 \%$ vs. $-34.5 \pm 9.5 \%, P<0.001$, Figure 9). HR was significantly reduced compared both to baseline and control animals in response to fasudil in diabetic animals $(P<0.05$, Figure 9).

\section{Vessel recruitment during fasudil treatment}

The number of vessels visible during ACh or SNPmediated vasodilation was not significantly different between control and diabetic rats. During combined NOS/ COX blockade vessel recruitment was significantly greater in diabetic animals compared to controls $(\triangle 19.9 \pm 10.8 \%$ vs. $-12.7 \pm 7.8 \%, P<0.05$, Figure 9 ). Following stimulation with $\mathrm{ACh}$, in the presence of combined blockade, this difference in recruitment was reduced. Vessel recruitment in response to fasudil was markedly increased in diabetic animals compared to baseline $(P<0.05)$, however this was not significantly different to control animals. 


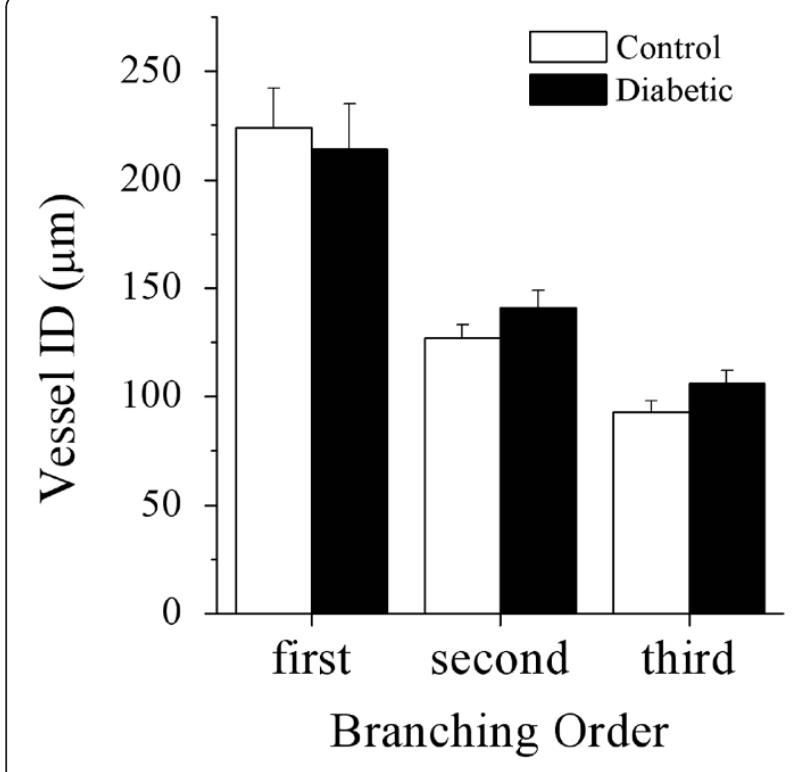

Figure 7 Vessel internal diameter categorised by branching order in control and diabetic animals during baseline vehicle infusion. There was no significant difference between control and diabetic animals. Control, $n=6$ and diabetic, $n=8$. Values expressed as mean $\pm \mathrm{SEM}$.

\section{Effect of fasudil on vascular constrictions}

One focal constriction ( $>70 \%$ of basal ID) was observed in a control and a diabetic rat (Figure 5F, white arrow) during combined blockade, but was abolished by ACh infusion in the control rat only. Subsequent fasudil treatment was able to completely eliminate the remaining focal constriction in the diabetic rat. Segmental constrictions were more prevalent in diabetic animals, following combined blockade (Figure 5F and 6E; white arrows and Figure 10), although this was not significant compared to control animals. Constrictions occurred predominantly in the first and second order vessels from diabetic animals (Figure 10). Consistent with the focal constriction findings, ACh administration during combined blockade completely eliminated all segmentally constricted vessels in control but not diabetic animals (Figure 10). Following fasudil treatment, segmental constrictions were markedly reduced in diabetic animals (Figures $5 \mathrm{H}$ and $6 \mathrm{G}$ and $\mathrm{H}$; black arrows) compared to NOS/COX blockade alone, notably in second and third order vessels.

\section{Discussion}

This study has demonstrated a widespread involvement of the ROCK signalling pathway in early stage diabetic coronary dysfunction in vivo. This is supported by data showing that acute ROCK inhibition alleviates focal and importantly segmental coronary constrictions in early diabetes. Furthermore, acute ROCK inhibition in the diabetic heart results in a significant increase in both micro- and macrovessel calibre in second and third order branching segments, following NOS/COX inhibition. This role for ROCK activation occurs prior to impairment in endothelium-dependent vasodilation, coronary structure or the ability of the coronary circulation to recruit microvessels during blood flow increases.

The three-week post diabetes induction time point used in this experiment, thus allows the investigation of early functional changes in the diabetic vasculature prior to changes in coronary structure, which are known to occur in the later stages of diabetes [24-26]. The extent of perivascular fibrosis, media-to-lumen ratio and capillary density were similar in diabetic and control animals and furthermore were consistent with our previous findings [6]. In addition, the likelihood that myogenic, metabolic or endothelial responses differentially affect coronary tone is limited, as coronary driving pressures were similar in diabetic and control animals during all treatments.

\section{Vascular function in the presence of nitric oxide and prostacyclin}

Basal coronary endothelial function in diabetic rats was maintained at 3 weeks post induction, however there are reports from experiments in isolated coronary arteries suggesting that by 4 weeks, endothelium-dependent vasodilation is impaired $[27,28]$. Consistent with the current findings, in our previous study, although there were early indications basal coronary function was starting to deteriorate after 3 weeks of diabetes [6], coronary vasodilation in vivo was actually maintained. This preserved coronary endothelium-dependent vasodilation, may be due to a compensatory increase in coronary COX-2 activity and expression as demonstrated in early stage diabetic mice [29]. It may also result from an increased expression of eNOS, which was found in carotid arteries in early diabetes, although this is less likely as the increase in activity is also associated with greater eNOS uncoupling [30]. Notably, vascular smooth muscle function in diabetic animals was well maintained as evidenced by similar endothelium-independent vasodilation when compared to control animals.

\section{Basal EDHF function and reserve}

Vasodilatory responses were similar post-NOS/COX blockade across all branching orders and vessel classes in diabetic and control animals although the ability of the diabetic coronary circulation to recruit new vessels was slightly enhanced. This increase in visible perfused segments likely results from an acute compensatory response to maintain coronary blood flow following NOS/ COX blockade. Endothelium stimulation following combined blockade facilitates the assessment of the ability of the remaining endogenous vasodilators namely, EDHF, 
A

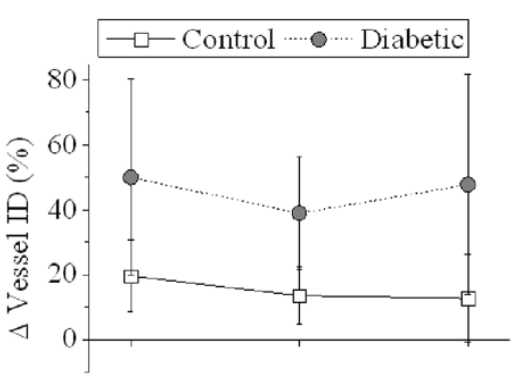

B

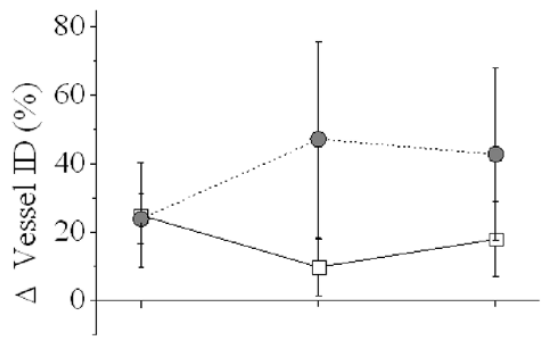

C

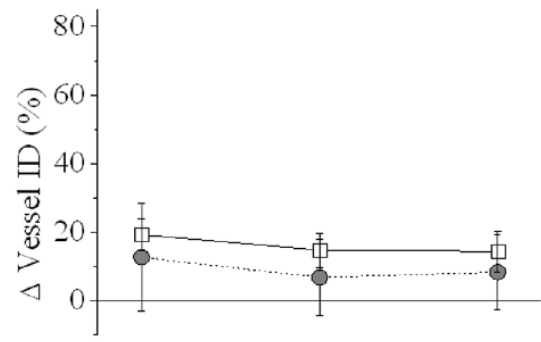

D

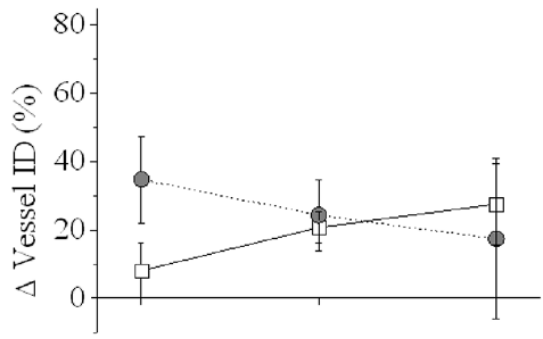

$\mathbf{E}$

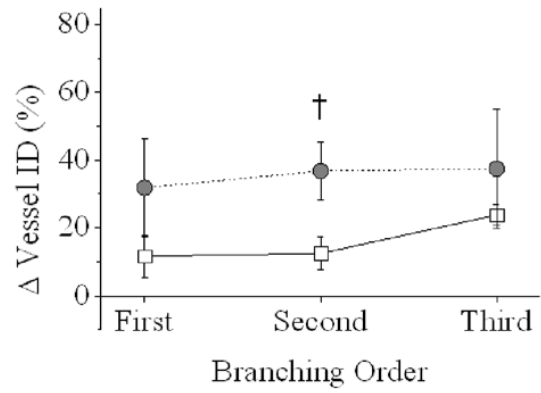

F

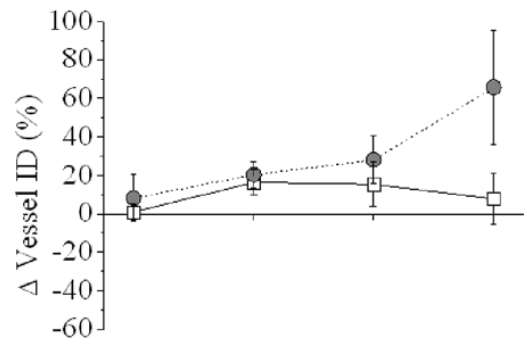

G

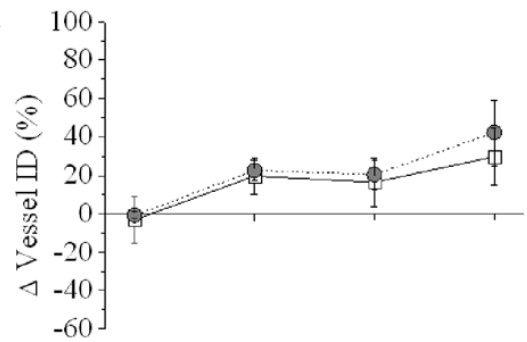

H

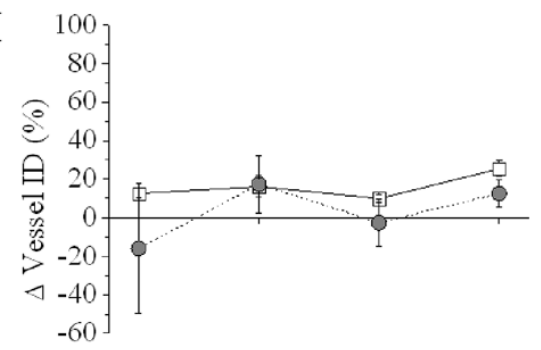

I

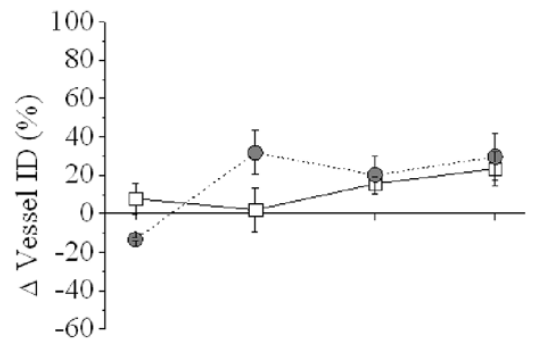

$\mathbf{J}$

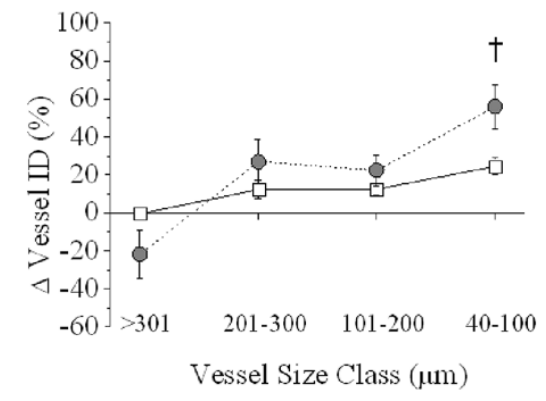

Figure 8 Change in vessel ID in control and diabetic animals during infusion of vasoactive compounds, categorised by branching order and vessel size class. ACh (A \& F; $3.0 \mu \mathrm{g} / \mathrm{kg} / \mathrm{min})$, SNP (B \& G; $3.0 \mu \mathrm{g} / \mathrm{kg} / \mathrm{min})$, combined (C \& H); L-NAME (10 mg/kg iv. bolus) + meclofenamate $(2 \mathrm{mg} / \mathrm{kg}$ iv. bolus), combined + ACh (D \& I) and combined + fasudil (E \& J; $10 \mathrm{mg} / \mathrm{kg}$ iv.). Fasudil treatment resulted in a marked increase in diabetic second order vessel ID and the 40-100 $\mu \mathrm{m}$ small artery-arterioles compared to controls. Control, $n=6$ and diabetic, $n=8$. Values expressed as mean \pm SEM. $+P<0.05$ vs. control. 


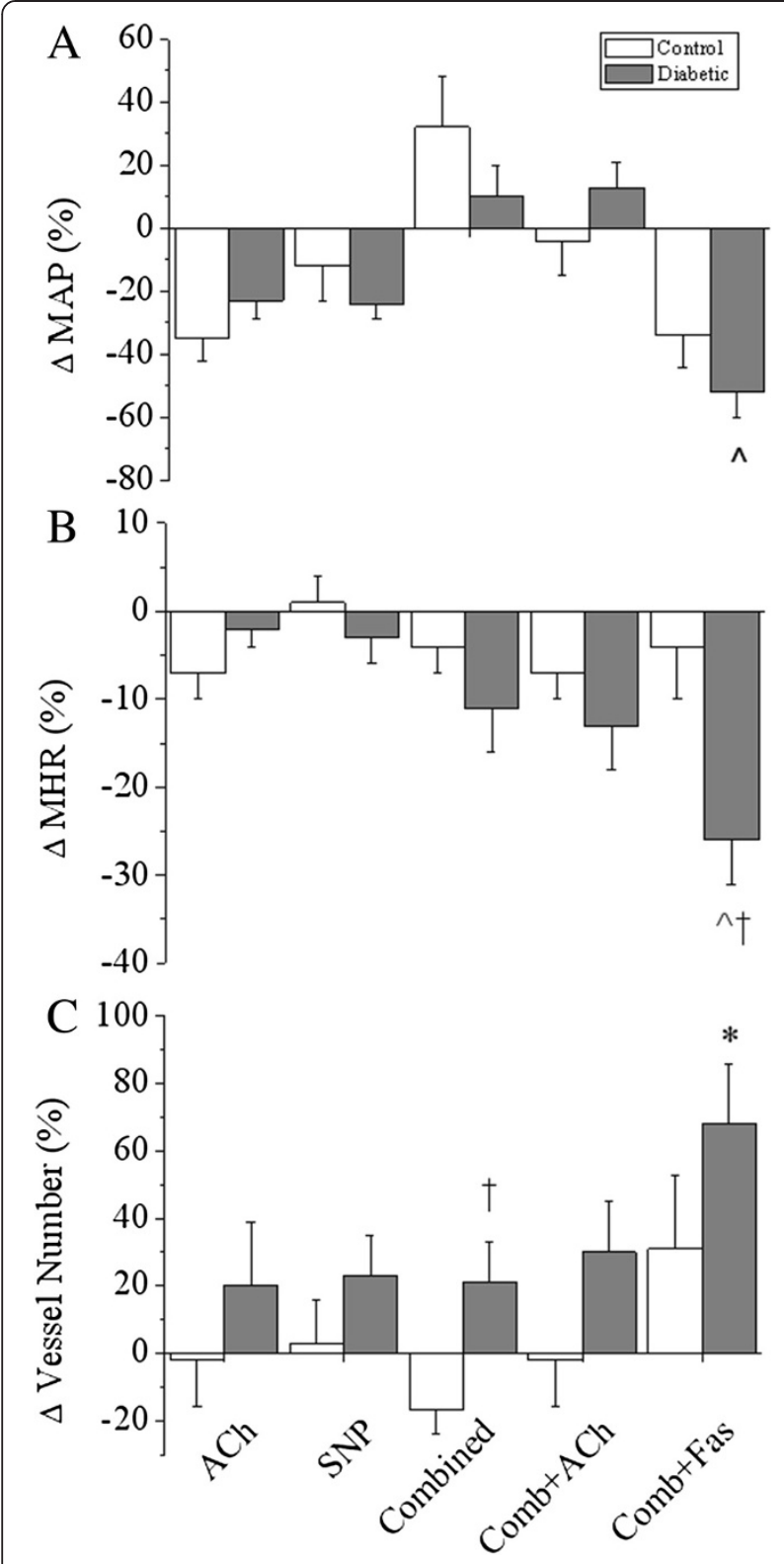

Figure 9 Mean arterial pressure (A), heart rate (B) and overall vessel recruitment $(C)$ in control and diabetic rats. Changes were assessed during infusion of ACh $(3.0 \mu \mathrm{g} / \mathrm{kg} / \mathrm{min})$, SNP $(3.0 \mu \mathrm{g} / \mathrm{kg} / \mathrm{min})$, combined; L-NAME (10 mg/kg iv. bolus) + meclofenamate $(2 \mathrm{mg} / \mathrm{kg}$ iv. bolus), combined + ACh and combined + fasudil (10 mg/kg iv.) relative to baseline (lactate $3.0 \mathrm{ml} / \mathrm{hr}$ ). A, Diabetic rats had a significant decrease in blood pressure during combined + fasudil treatment compared to baseline. $\mathbf{B}$, Heart rate was significantly reduced in diabetic animals during fasudil treatment compared to baseline and control rats. C, Diabetic rats had significantly greater vessel recruitment during combined NOS/COX blockade compared to control animals. Control, $n=6$ and diabetic, $n=8$. Values expressed as mean $\pm S E M$. ${ }^{*} P<0.05, \wedge P<0.001$ vs. baseline. $+P<0.05$ vs. control.

to maintain vasodilation. These findings agree with our previous study [6], in suggesting that global coronary EDHF reserve is unchanged in this model of diabetes, as EDHF

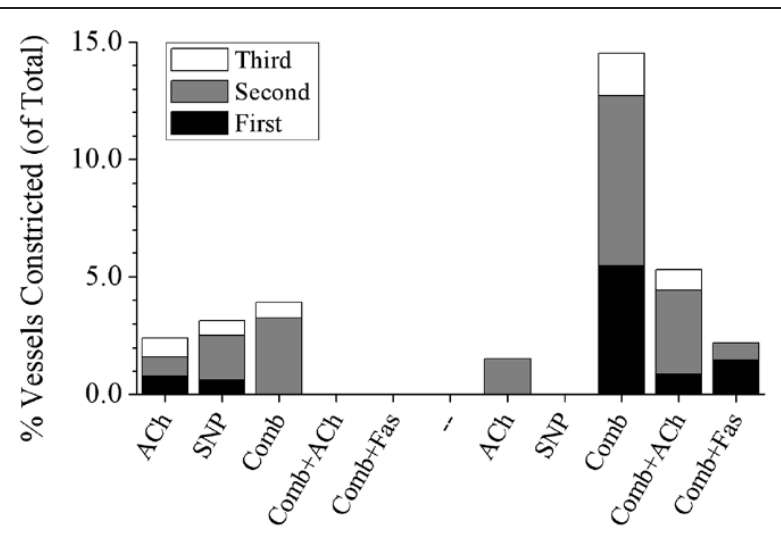

Control

Diabetic

Figure 10 The occurrence of segmental constrictions classified by branching order in control and diabetic rats. Assessed during administration of ACh $(3.0 \mu \mathrm{g} / \mathrm{kg} / \mathrm{min})$, SNP $(3.0 \mu \mathrm{g} / \mathrm{kg} / \mathrm{min})$, combined; L-NAME (10 mg/kg iv. bolus) + meclofenamate $(2 \mathrm{mg} / \mathrm{kg}$ iv. bolus), combined + ACh and combined + fasudil (10 mg/kg iv.). Segmental constrictions predominately occurred in second order vessels in control animals. In diabetic animals constrictions were predominant in second order vessels and to a lesser extent in first order. ACh administration following combined NOS/COX blockade alleviated first order constrictions while fasudil treatment further reduced second order constrictions. Segmental constrictions are expressed as a proportion of total vessels visible. Control, $n=6$ and diabetic, $n=8$. Values expressed as mean \pm SEM.

pathways remain intact and capable of providing sufficient reserve to overcome any vasoconstrictor influences.

\section{Vessel diameter response to fasudil}

To assess the role of ROCK in the diabetic coronary circulation in vivo, this study utilised the potent and selective ROCK inhibitor fasudil, due to its minimal off-target effects on related signalling pathways, including myosin light chain kinase and protein kinase $\mathrm{C}(\mathrm{PKC})$ [31]. Fasudil is non-selective for the isoforms of ROCK, ROCK1, expressed ubiquitously, except in brain and muscle tissue and ROCK2, expressed primarily in cardiac and brain tissue [32]. However, it is unlikely that the limited time of fasudil treatment in the current study would be sufficient to result in its significant conversion to the more highly potent and selective, hydroxyfasudil [18] and as such some of the inhibitory effect of fasudil may result in part from off-target inhibition of other kinases. Importantly, in the context of cardiovascular disease and diabetic coronary dysfunction, both ROCK1 and ROCK2 are expressed in vascular endothelial and smooth muscle cells [33-35].

In the diabetic coronary microcirculation ROCK inhibition resulted in significantly greater vasodilation compared to controls that was most evident in the small arteries and arterioles emerging from the epicardial main artery segments. Hence, acute fasudil treatment increases global perfusion in the early diabetic heart. Consistent with these 
findings, Didion et al. have shown that ROCK inhibition significantly increased vessel diameter of cerebral arteries from type-2 diabetic $\mathrm{db} / \mathrm{db}$ mice but not in controls [20]. Moreover, fasudil has been shown to reverse vasoconstriction in more advanced diabetes $[33,36]$.

\section{Focal and segmental constrictions in diabetes}

In the early diabetic state, prior to widespread vessel impairment in coronary endothelium mediated vasodilatation, we have characterised and quantified focal and segmental constrictions in the microvasculature [6]. This study also revealed a strong trend towards an increased incidence of segmental constrictions in diabetic animals when there is a deficit in vasodilator production. This suggests that in later stage diabetes, when the actions of $\mathrm{NO}$ and $\mathrm{PGI}_{2}$ are progressively impaired [37], the coronary circulation may become more vulnerable to constrictions. Therefore, further assessment of the role these constrictions play in the regulation of coronary perfusion in diabetes remains vital.

Endothelium-dependent stimulation post-NOS/COX blockade abolished all segmental constrictions in control but not diabetic animals. More detailed analysis showed that the segmental constrictions occurred predominantly in epicardial first order vessels in diabetic animals. This is somewhat unexpected as small alterations in vascular tone, at this macrovessel level are less common, as they can have significant effects on downstream microvascular perfusion and are actually predictive of longer term cardiovascular disease risk in type-2 diabetes [38].

\section{A role for ROCK in focal and segmental constrictions}

A novel finding in this study, is the in vivo identification of ROCK as a likely mediator of localised constrictions in diabetic hearts. This is supported by the fact that acute ROCK inhibition greatly reduced the incidence of segmental constrictions following NOS/COX blockade. Consistent with this, ROCK has previously been shown to be upregulated in non-diabetic porcine hearts at the site of coronary artery spasm [39]. Hypercontraction by ROCK activation and increased myosin binding subunit phosphorylation in vascular smooth muscle cells appears to be a key to much vascular dysfunction. Localised ROCK activation has been implicated in cerebral artery vasospasm following subarachnoid haemorrhage [40], coronary artery spasm [10], and following coronary artery bypass [41] and myocardial ischemia [17]. In our study, nonconstricted vascular regions in diabetic animals responded to ROCK inhibition similarly to that of responses in control animals, in agreement with findings in the human coronary circulation where ROCK inhibition had minimal effect on non-spastic segments [42]. This suggests that during early-stage diabetes there remain vessel segments where changes in ROCK signalling are not contributing appreciably to vasomotor responses.
Immunostaining for ROCK1 expression in the myocardium and endothelium was not significantly different between diabetic and control animals although there was a notable trend towards an increase. While not significant, our results are in line with previous work which showed significant ROCK1 expression upregulation in thoracic aortas from 3-week diabetic rats [43]. ROCK2 expression was borderline significantly increased in our diabetic animals, as reported in retinal vessels from rats 2 weeks after inducing diabetes [15]. Irrespective of ROCK expression levels in the early diabetic heart a functional role for ROCK activation was clear.

Diabetes is known to increase RhoA expression, the upstream regulator of ROCK, as shown in the basilar artery membrane at 2 weeks [44] and aortic homogenates at 4-weeks post STZ [28] in diabetic rodents. Furthermore, RhoA expression remains elevated in more advanced diabetic stages, as described in aorta from 12-14 week old [13] and 12-40 week old [33] $\mathrm{db} / \mathrm{db}$ mice. However, to date the effect of diabetes on coronary ROCK expression and activity in more advanced stages remain unclear. Longer term type-2 diabetes studies using aortic and mesenteric arteries suggest that ROCK expression is unchanged $[33,36]$, although whether this is true in the coronary circulation is uncertain. It remains to be determined if RhoA upregulation drives ROCK activation and localised coronary constrictions in early diabetes.

\section{Possible mechanisms for ROCK-mediated vasoconstriction}

There is strong evidence that hyperglycaemia and or hyperlipidaemia evoke reactive oxygen species to stimulate RhoA/Rho-kinase signalling [45]. Remnant lipoproteins, acting via the RhoA/Rho-kinase pathway to cause coronary vasospasm are considered to be a major factor in sudden death in humans [46]. Nevertheless, our findings suggests that lipids may have a minimal role in the initiation of ROCK-mediated vasoconstriction in our diabetic animals, as plasma triglyceride concentrations were comparable to control animals, contrasting with later diabetic states $[27,47]$. Furthermore, the absence of a significant widespread increase in nitrotyrosine levels in the coronary vessels and myocardium of diabetic rats in this study suggests that oxidative stress is not yet contributing appreciably to ROCK-mediated vasoconstriction at this time point. Plasma triglycerides and nitrotyrosine are only single measures of lipid accumulation and oxidative stress, therefore further assessment of lipid profiles and other reactive oxygen and nitrogen species are certainly warranted.

Hyperglycaemia and diacylglycerol accumulation are well known for inducing activation of multiple PKC isoforms, and in particular $\mathrm{PKC} \beta$, to cause vascular complications and potentiated vasoconstrictor responses in diabetic coronary arteries [48]. While PKC mediated potentiation of $\mathrm{ET}_{1}$ constrictor tone is implicated, diabetic myocardial 
contractile impairment has been shown to be a consequence of PKC activation of downstream inducible NOS/ RhoA/ROCK [49-51]. Kizub et al. also suggest that both ROCK and PKC activity evoke enhanced myofilament $\mathrm{Ca}^{2+}$ sensitivity in muscular arteries in diabetes [52]. It is important to note however that ROCK inhibition did not completely alleviate all diabetic segmental constrictions, suggesting that although important, ROCK is unlikely to be the sole mediator of the elevated diabetic coronary constrictor tone.

\section{Experimental limitations}

In comparison to our previous study of early diabetes [6], the extent of microvascular dysfunction, both focal and segmental constriction, was slightly less advanced in this cohort of diabetic animals. This was also the case with the attenuation of endothelium-dependent vessel recruitment, which in this study, was not significantly different from controls. This may be due to a slower development in the pathogenesis of diabetes. Indeed, although the average blood glucose concentration was greater in the current study, this was also coupled with greater between animal variability in BG (12.7- $27.9 \mathrm{mmol} / \mathrm{L}$ this study vs. 16.7-19.4 mmol/L) [6]. Some diabetic animals in the current study may have initially had lower blood glucose concentrations for part of the 3-week period after STZ induction. This could have led to a shortened period of severe hyperglycaemia and the partial maintenance of coronary microvascular function.

A second limitation of this study that needs to be recognised is that we cannot accurately determine global coronary flow or velocity in the anaesthetised rat heart at this time with the SATICON detector video frame rates [53]. The advantages of SR microangiography, including increased spatial and temporal resolution, far outweigh this limitation, and allows the assessment of coronary microvessel diameter, and therefore local distribution of vascular resistance and function in vivo $[6,53]$.

\section{Future directions}

Future investigations may focus on treating diabetic cardiomyopathy with ROCK inhibitors. Indeed recent evidence in diabetic rodents suggests that acute ROCK inhibition also corrects myocardial contractile function $[49,54]$. Fasudil treatment has also been shown to preserve the post-conditioning capacity of the heart during hyperglycaemia and protect against myocardial infarction through the opening of mitochondrial ATP-sensitive K channels [55]. Furthermore, others have shown that inhibition of ROCK reduces monocyte cell adhesion to the endothelium [56], an early event in plaque formation, and reduces early plaque formation and the size of established plaques [57]. Since ROCK is an early contributor to coronary vascular dysfunction, by increasing constrictor tone and subsequently endothelial dysfunction, then chronic treatment with ROCK inhibitors may help prevent the diabetic coronary dysfunction completely. A potential added benefit of this therapy is likely to be a reduction in diabetic vascular complications associated with both vascular inflammation and the pro-atherogenic state in diabetics [56].

\section{Conclusions}

In summary, we have confirmed that in vivo imaging using $\mathrm{SR}$ is a powerful means of investigating the intact coronary circulation. Furthermore, we have shown in vivo, that in early-diabetic rats increased ROCK activity plays a role in coronary microvasculature function, as acute ROCK inhibition increased coronary perfusion. Notably, ROCKmediated vasoconstriction was not a consequence of increased oxidative stress, inferring that it was most likely mediated by increased activation of myosin light chain phosphatase. However, at this early diabetic stage the ROCK mediated increase in vasoconstrictor tone is normally offset by $\mathrm{NO}$ and $\mathrm{PGI}_{2}$. Nevertheless, these findings highlight the potential importance of the ROCK signalling pathway in diabetic coronary vasculature and provide a promising target for future therapeutic interventions.

\section{Abbreviations}

ACh: Acetylcholine; BG: Blood glucose; BW: Body weight; COX: Cyclo-oxygenase; EDHF: Endothelium derived hyperpolarising factors; eNOS: Endothelial nitric oxide synthase; $\mathrm{ET}_{1}$ : Endothelin 1; HR: Heart rate; ID: Internal diameter; L-NAME: N $\omega$-nitro-l-arginine methyl ester; LV: Left ventricle; MAP: Mean arterial pressure; NO: Nitric oxide; NOS: Nitric oxide synthase; $\mathrm{PGI}_{2}$ : Prostacyclin; PKC: Protein kinase C; ROCK: RhoA/Rho-kinase; SEM: Standard error of the mean; SNP: Sodium nitroprusside; SR: Synchrotron radiation; STZ: Streptozotocin.

\section{Competing interests}

The authors declare that they have no competing interests.

\section{Authors' contributions}

JTP, MJJ and MS participated in the design of the study, carried out the imaging experiments and contributed to the data analysis and writing of the manuscript. AJE participated in the imaging studies, oversaw and optimised immunohistochemistry. TS, MJJ, DOS, YF and HT prepared animals and collected physiological data for the imaging experiments. MJJ, MW and DJK contributed to histology and immunohistochemistry preparation and analysis and supervision of these analyses. All authors read and approved the final manuscript.

\section{Acknowledgements}

Experiments were conducted at SPring-8, Japan Synchrotron Radiation Research Institute, Hyogo, Japan (Proposals 2009A1467, 2010A1260, 2010B1372) with approval from the Animal Experiment Review Committee. DJK was supported by a National Health and Medical Research Council Senior Research Fellowship. The authors acknowledge support from the International Synchrotron Access Programme (IA104) managed by the Australian Synchrotron and funded by the Australian Government, Intramural Research Funds (22-2-3, 22-3-2) of the National Cerebral and Cardiovascular Center, Grant-in-Aid from the Ministry of Education, Culture, Sports, Science and Technology of Japan (16659210, 20590242, 23249038, 23650213). The authors are grateful to Kath MacLeod for comments on an earlier draft of this manuscript. 


\section{Author details}

'Department of Physiology, Monash University, Melbourne, Australia. ${ }^{2}$ Monash Biomedical Imaging Facility, Melbourne, Australia. ${ }^{3}$ Australian Synchrotron, Melbourne, Australia. ${ }^{4}$ Department of Medicine, St Vincent's Hospital, University of Melbourne, Melbourne, Australia. ${ }^{5}$ National Cerebra and Cardiovascular Center Research Institute, Suita, Japan. ${ }^{6}$ The Ritchie Centre, Monash Institute of Medical Research, Melbourne, Australia. Department of Physiology, Otago University, Dunedin, New Zealand.

${ }^{8}$ Japan Synchrotron Radiation Research Institute, Harima, Japan.

Received: 29 July 2013 Accepted: 30 July 2013

Published: 1 August 2013

\section{References}

1. Prior JO, Quiñones MJ, Hernandez-Pampaloni M, Facta AD, Schindler TH, Sayre JW, Hsueh WA, Schelbert HR: Coronary circulatory dysfunction in insulin resistance, impaired glucose tolerance, and type 2 diabetes mellitus. Circulation 2005, 111(18):2291-2298.

2. Nahser PJ Jr, Brown RE, Oskarsson H, Winniford MD, Rossen JD: Maximal coronary flow reserve and metabolic coronary vasodilation in patients with diabetes mellitus. Circulation 1995, 91(3):635-640

3. Yonaha O, Matsubara T, Naruse K, Ishii H, Murohara T, Nakamura J, Amano T, Hotta N: Effects of reduced coronary flow reserve on left ventricular function in type 2 diabetes. Diabetes Res Clin Pract 2008, 82(1):98-103.

4. Almdal T, Scharling $H$, Jensen JS, Vestergaard $H$ : The independent effect of type 2 diabetes mellitus on ischemic heart disease, stroke, and death: A population-based study of 13000 men and women with 20 years of follow-up. Arch Intern Med 2004, 164(13):1422-1426.

5. Nathan DM: Long-term complications of diabetes mellitus. New Eng/ J Med 1993, 328(23):1676-1685.

6. Jenkins MJ, Edgley AJ, Sonobe T, Umetani K, Schwenke DO, Fujii Y, Brown RD, Kelly DJ, Shirai M, Pearson JT: Dynamic synchrotron imaging of diabetic rat coronary microcirculation in vivo. Arterioscler Thromb Vasc Biol 2012, 32(2):370-377.

7. Mukai Y, Shimokawa H, Matoba T, Kandabashi T, Satoh S, Hiroki J, Kaibuchi K, Takeshita A: Involvement of Rho-kinase in hypertensive vascular disease: a novel therapeutic target in hypertension. FASEB J 2001, 15(6):1062-1064

8. Shibuya M, Hirai S, Seto M, Satoh SI, Ohtomo E: Effects of fasudil in acute ischemic stroke: Results of a prospective placebo-controlled doubleblind trial. J Neuro/ Sci 2005, 238(1-2):31-39.

9. Masumoto A, Mohri M, Shimokawa H, Urakami L, Usui M, Takeshita A: Suppression of coronary artery spasm by the Rho-kinase inhibitor fasudil in patients with vasospastic angina. Circulation 2002, 105(13):1545-1547.

10. Kandabashi T, Shimokawa H, Miyata K, Kunihiro I, Eto Y, Morishige K, Matsumoto Y, Obara K, Nakayama K, Takahashi S, et al: Evidence for protein kinase C-mediated activation of Rho-kinase in a porcine model of coronary artery spasm. Arterioscler Thromb Vasc Biol 2003, 23(12):2209-2214.

11. Kishi T, Hirooka Y, Masumoto A, Ito K, Kimura Y, Inokuchi K, Tagawa T, Shimokawa H, Takeshita A, Sunagawa K: Rho-kinase inhibitor improves increased vascular resistance and impaired vasodilation of the forearm in patients with heart failure. Circulation 2005, 111(21):2741-2747.

12. Hamid SA, Bower HS, Baxter GF: Rho kinase activation plays a major role as a mediator of irreversible injury in reperfused myocardium. Am J Physiol Heart Circ Physiol 2007, 292(6):H2598-H2606.

13. Xie Z, Su W, Guo Z, Pang H, Post SR, Gong MC: Up-regulation of CPI-17 phosphorylation in diabetic vasculature and high glucose cultured vascular smooth muscle cells. Cardiovasc Res 2006, 69(2):491-501.

14. Noma K, Oyama N, Liao JK: Physiological role of ROCKs in the cardiovascular system. Am J Physiol Cell Physiol 2006, 290(3):C661-C668.

15. Arita R, Hata Y, Nakao S, Kita T, Miura M, Kawahara S, Zandi S, Almulki L, Tayyari F, Shimokawa $H$, et al: Rho kinase inhibition by fasudil ameliorates diabetes-induced microvascular damage. Diabetes 2009, 58(1):215-226.

16. Budzyn K, Marley PD, Sobey CG: Targeting Rho and Rho-kinase in the treatment of cardiovascular disease. Trends Pharmacol Sci 2006, 27(2):97-104.

17. Mohri M, Shimokawa H, Hirakawa Y, Masumoto A, Takeshita A: Rho-kinase inhibition with intracoronary fasudil prevents myocardial ischemia in patients with coronary microvascular spasm. J Am Coll Cardiol 2003, 41(1):15-19.

18. Satoh SI, Utsunomiya T, Tsurui K, Kobayashi T, Ikegaki I, Sasaki Y, Asano T: Pharmacological profile of hydroxy fasudil as a selective rho kinase inhibitor on ischemic brain damage. Life Sci 2001, 69(12):1441-1453.
19. Yada T, Shimokawa H, Hiramatsu O, Kajita T, Shigeto F, Tanaka E, Shinozaki Y, Mori H, Kiyooka T, Katsura M, et al: Beneficial effect of hydroxyfasudil, a specific Rho-kinase inhibitor, on ischemia/reperfusion injury in canine coronary microcirculation in vivo. J Am Coll Cardiol 2005, 45(4):599-607.

20. Didion SP, Lynch CM, Baumbach GL, Faraci FM: Impaired endotheliumdependent responses and enhanced influence of Rho-kinase in cerebral arterioles in type II diabetes. Stroke 2005, 36(2):342-347.

21. Kobayashi N, Horinaka S, Mita SI, Nakano S, Honda T, Yoshida K, Kobayashi T, Matsuoka $\mathrm{H}$ : Critical role of Rho-kinase pathway for cardiac performance and remodeling in failing rat hearts. Cardiovasc Res 2002, 55(4):757-767.

22. Schwenke DO, Pearson JT, Sonobe T, Ishibashi-Ueda H, Shimouchi A Kangawa K, Umetani K, Shirai M: Role of Rho-kinase signaling and endothelial dysfunction in modulating blood flow distribution in pulmonary hypertension. J App/ Physio/ 2011, 110(4):901-908.

23. Hagikura K, Fukuda N, Yokoyama S, Yuxin L, Kusumi Y, Matsumoto T, Ikeda $Y$, Kunimoto S, Takayama T, Jumabay M: Low invasive angiogenic therapy for myocardial infarction by retrograde transplantation of mononuclear cells expressing the VEGF gene. Int J Cardiol 2010, 142(1):56-64.

24. Fiordaliso F, Cuccovillo I, Bianchi R, Bai A, Doni M, Salio M, De Angelis N, Ghezzi P, Latini R, Masson S: Cardiovascular oxidative stress is reduced by an ACE inhibitor in a rat model of streptozotocin-induced diabetes. Life Sci 2006, 79(2):121-129.

25. Patumraj S, Tewit S, Amatyakul S, Jariyapongskul A, Maneesri S, Kasantikul V, Shepro D: Comparative effects of garlic and aspirin on diabetic cardiovascular complications. Drug Deliv 2000, 7(2):91-96.

26. Jesmin S, Zaedi S, Shimojo N, lemitsu M, Masuzawa K, Yamaguchi N Mowa CN, Maeda S, Hattori Y, Miyauchi T: Endothelin antagonism normalizes VEGF signaling and cardiac function in STZ-induced diabetic rat hearts. Am J Physiol Endocrinol Metab 2007, 292(4):E1030-E1040.

27. Tawfik HE, El-Remessy AB, Matragoon S, Ma G, Caldwell RB, Caldwell RW: Simvastatin improves diabetes-induced coronary endothelial dysfunction. J Pharmacol Exp Ther 2006, 319(1):386-395.

28. El-Remessy AB, Tawfik HE, Matragoon S, Pillai B, Caldwell RB, Caldwell RW: Peroxynitrite mediates diabetes-induced endothelial dysfunction: possible role of Rho kinase activation. Exp Diabetes Res 2010, 2010:247861.

29. Nacci C, Tarquinio M, De Benedictis L, Mauro A, Zigrino A, Carratù MR, Quon MJ, Montagnani M: Endothelial dysfunction in mice with streptozotocininduced type 1 diabetes is opposed by compensatory overexpression of cyclooxygenase-2 in the vasculature. Endocrinology 2009, 150(2):849-861.

30. Leo $\mathrm{CH}$, Joshi $\mathrm{A}$, Woodman OL: Short-term type 1 diabetes alters the mechanism of endothelium-dependent relaxation in the rat carotid artery. Am J Physiol Heart Circ Physiol 2010, 299(2):H502-H511.

31. Shimokawa H, Seto M, Katsumata N, Amano M, Kozai T, Yamawaki T, Kuwata K, Kandabashi T, Egashira K, Ikegaki I, et al: Rho-kinase-mediated pathway induces enhanced myosin light chain phosphorylations in a swine model of coronary artery spasm. Cardiovasc Res 1999, 43(4):1029-1039.

32. Nakagawa O, Fujisawa K, Ishizaki T, Saito Y, Nakao K, Narumiya S: ROCK-I and ROCK-II, two isoforms of Rho-associated coiled-coil forming protein serine/threonine kinase in mice. FEBS Lett 1996, 392(2):189-193.

33. Nuno DW, Harrod JS, Lamping KG: Sex-dependent differences in Rho activation contribute to contractile dysfunction in type 2 diabetic mice. Am J Physiol Heart Circ Physiol 2009, 297(4):H1469-H1477.

34. Wibberley A, Chen Z, Hu E, Hieble JP, Westfall TD: Expression and functional role of Rho-kinase in rat urinary bladder smooth muscle. $\mathrm{Br} J$ Pharmacol 2003, 138(5):757-766.

35. Ming XF, Barandier C, Viswambharan H, Kwak BR, Mach F, Mazzolai L, Hayoz D, Ruffieux J, Rusconi S, Montani JP, et al: Thrombin stimulates human endothelial arginase enzymatic activity via RhoA/ROCK pathway: Implications for atherosclerotic endothelial dysfunction. Circulation 2004 110(24):3708-3714.

36. Matsumoto T, Kobayashi T, Ishida K, Taguchi K, Kamata K: Enhancement of mesenteric artery contraction to 5-HT depends on Rho kinase and Src kinase pathways in the ob/ob mouse model of type 2 diabetes. $\mathrm{Br} J$ Pharmacol 2010, 160(5):1092-1104.

37. Zou MH, Cohen RA, Ullrich V: Peroxynitrite and vascular endothelial dysfunction in diabetes mellitus. Endothelium 2004, 11(2):89-97.

38. Nitenberg A, Valensi P, Sachs R, Cosson E, Attali JR, Antony I: Prognostic value of epicardial coronary artery constriction to the cold pressor test in Type 2 diabetic patients with angiographically normal coronary arteries and no other major coronary risk factors. Diabetes Care 2004, 27(1):208-215. 
39. Kandabashi T, Shimokawa H, Miyata K, Kunihiro I, Kawano Y, Fukata Y, Higo T, Egashira K, Takahashi S, Kaibuchi K, et al: Inhibition of myosin phosphatase by upregulated Rho-kinase plays a key role for coronary artery spasm in a porcine model with interleukin-1 $\beta$. Circulation 2000, 101(11):1319-1323.

40. Sato M, Tani E, Fujikawa $H$, Kaibuchi $K$ : Involvement of Rho-kinase -mediated phosphorylation of myosin light chain in enhancement of cerebral vasospasm. Circ Res 2000, 87(3):195-200.

41. Inokuchi K, Ito A, Fukumoto Y, Matoba T, Shiose A, Nishida T, Masuda M, Morita S, Shimokawa H: Usefulness of fasudil, a Rho-kinase inhibitor, to treat intractable severe coronary spasm after coronary artery bypass surgery. J Cardiovasc Pharmacol 2004, 44(3):275-277.

42. Matsumoto T, Kajiya F: Coronary microcirculation: Physiology and mechanics. Fluid Dynam Res 2005, 37(1-2 SPEC. ISS):60-81.

43. Failli P, Alfarano C, Franchi-Micheli S, Mannucci E, Cerbai E, Mugelli A, Raimondi L: Losartan counteracts the hyper-reactivity to angiotensin II and ROCK1 over-activation in aortas isolated from streptozotocininjected diabetic rats. Cardiovasc Diabetol 2009, 8:32.

44. Miao L, Calvert JW, Tang J, Zhang JH: Upregulation of small GTPase RhoA in the basilar artery from diabetic (mellitus) rats. Life Sci 2002, 71(10):1175-1185.

45. Bailey SR, Mitra S, Flavahan S, Flavahan NA: Reactive oxygen species from smooth muscle mitochondria initiate cold-induced constriction of cutaneous arteries. Am J Physiol Heart Circ Physiol 2005, 289(1 58-1):H243-H250.

46. Oi K, Shimokawa H, Hiroki J, Uwatoku T, Abe K, Matsumoto Y, Nakajima Y, Nakajima K, Takeichi S, Takeshita A: Remnant lipoproteins from patients with sudden cardiac death enhance coronary vasospastic activity through upregulation of Rho-kinase. Arterioscler Thromb Vasc Biol 2004, 24(5):918-922.

47. Zhang FL, Ye C, Li G, Ding W, Zhou W, Zhu H, Chen G, Luo T, Guang M, Liu $Y$, et al: The rat model of type 2 diabetic mellitus and its glycometabolism characters. Exp Anim 2003, 52(5):401-407.

48. Tickerhoof MM, Farrell PA, Korzick DH: Alterations in rat coronary vasoreactivity and vascular protein kinase $\mathrm{C}$ isoforms in Type 1 diabetes. Am J Physiol Heart Circ Physiol 2003, 285:H2694-H2703.

49. Lin G, Craig GP, Zhang L, Yuen VG, Allard M, McNeill JH, MacLeod KM: Acute inhibition of Rho-kinase improves cardiac contractile function in streptozotocin-diabetic rats. Cardiovasc Res 2007, 75(1):51-58.

50. Nagareddy PR, Soliman H, Lin G, Rajput PS, Kumar U, McNeill JH, MacLeod KM: Selective Inhibition of Protein Kinase C $\beta 2$ Attenuates Inducible Nitric Oxide Synthase-Mediated Cardiovascular Abnormalities in Streptozotocin-Induced Diabetic Rats. Diabetes 2009, 58(10):2355-2364.

51. Soliman H, Craig GP, Nagareddy P, Yuen VG, Lin G, Kumar U, McNeill JH, MacLeod KM: Role of inducible nitric oxide synthase in induction of RhoA expression in hearts from diabetic rats. Cardiovasc Res 2008, 79(2):322-330.

52. Kizub I, Pavlova O, Johnson C, Soloviev A, Zholos A: Rho kinase and protein kinase $C$ involvement in vascular smooth muscle myofilament calcium sensitization in arteries from diabetic rats. Br J Pharmacol 2010, 159(8):1724-1731.

53. Shirai M, Schwenke DO, Eppel GA, Evans RG, Edgley AJ, Tsuchimochi H, Umetani K, Pearson JT: Synchrotron-based angiography for investigation of the regulation of vasomotor function in the microcirculation in vivo. Clin Exp Pharmacol Physiol 2009, 36(1):107-116.

54. Zhou H, Li YJ, Wang M, Zhang LH, Guo BY, Zhao ZS, Meng FL, Deng YG, Wang RY: Involvement of RhoA/ROCK in myocardial fibrosis in a rat model of type 2 diabetes. Acta Pharmacol Sin 2011, 32(8):999-1008.

55. Ichinomiya T, Cho S, Higashijima U, Matsumoto S, Maekawa T, Sumikawa K High-dose fasudil preserves postconditioning against myocardial infarction under hyperglycemia in rats: role of mitochondrial KATP channels. Cardiovasc Diabetol 2012, 11:28.

56. Li H, Peng W, Jian W, Li Y, Li Q, Li W, Xu Y: ROCK inhibitor fasudil attenuated high glucose-induced MCP-1 and VCAM-1 expression and monocyte-endothelial cell adhesion. Cardiovasc Diabetol 2012, 11:65.

57. Wu DJ, Xu JZ, Wu YJ, Jean-Charles L, Xiao B, Gao PJ, Zhu DL: Effects of fasudil on early atherosclerotic plaque formation and established lesion progression in apolipoprotein E-knockout mice. Atherosclerosis 2009, 207(1):68-73.

doi:10.1186/1475-2840-12-111

Cite this article as: Pearson et al: Acute Rho-kinase inhibition improves coronary dysfunction in vivo, in the early diabetic microcirculation. Cardiovascular Diabetology 2013 12:111.

\section{Submit your next manuscript to BioMed Central and take full advantage of:}

- Convenient online submission

- Thorough peer review

- No space constraints or color figure charges

- Immediate publication on acceptance

- Inclusion in PubMed, CAS, Scopus and Google Scholar

- Research which is freely available for redistribution 